\title{
The relation between gap discrimination and auditory stream segregation
}

\author{
DONNA L. NEFF and WALT JESTEADT \\ Boys Town Institute for Communication Disorders in Children, Omaha, Nebraska \\ and \\ EVAN L. BROWN \\ University of Nebraska at Omaha, Omahä, Nebraska
}

\begin{abstract}
Gap discrimination and stream segregation were examined using sequences of $2,4,8$, or 16 tones. The frequency differences between tones ranged from $1 / 24$ to $2 \frac{1}{2}$ octaves. Judgments of stream segregation show large intersubject variability, whereas gap thresholds are comparatively stable. Gap thresholds and streaming judgments are both affected by the frequency separation between tones. However, only streaming judgments are affected by presentation rate. Gap-threshold functions show no discontinuities or plateaus with increasing frequency differences and faster presentation rates. These results suggest that stream segregation is not a primary factor limiting gap-discrimination performance in tonal sequences.
\end{abstract}

Stream segregation (SS) refers to a change in the perceptual organization of a sequence of sounds. As various stimulus parameters change, a single coherent series of sounds seems to split perceptually into concurrent subsets of related sounds (Bregman \& Campbell, 1971). This perceptual change has been observed most frequently with tonal sequences containing widely disparate frequencies (e.g., Bregman, 1978c; Bregman \& Campbell, 1971; Bregman \& Rudnicky, 1975; van Noorden, 1975). Gap discrimination is one of several procedures used to assess the temporal resolving power of the auditory system. In a gap-discrimination task, the listener is asked to detect a difference in the duration of a silent interval or gap between marker stimuli. In general, performance in gap discrimination deteriorates as the frequency difference between tonal markers surrounding the gap is increased for both two-tone (e.g., Williams \& Perrott, 1972) and longer sequences (e.g., van Noorden, Note 1). Although SS has been invoked on occasion to help explain these results (Divenyi \& Danner, 1977; Divenyi \& Sachs, 1978; Fitzgibbons, Pollatsek, \& Thomas, 1974), the relation between SS and gap discrimination has not been established.

\section{Auditory Stream Segregation}

Stream segregation, as proposed by Bregman and

We thank John Flowers, Charles Watson, and Daniel Weber for their comments on earlier drafts. This research was supported by NIH Grant NS-14709. A portion of the research described in this paper was presented at the 98th meeting of the Acoustical Society of America. Address correspondence to Donna L. Neff, Human Communication Laboratories, Boys Town Institute, 555 N. 30 St., Omaha, Nebraska 68131. his colleagues (Bregman, 1978a, 1978b, 1978c, 1978d; Bregman \& Campbell, 1971; Bregman \& Dannenbring, 1973; Bregman \& Rudnicky, 1975; Dannenbring \& Bregman, 1976a), reflects a parsing mechanism that organizes acoustic input according to the implied source of the sound. For SS based on frequency, the major parameters affecting perceptual organization are the frequency separation of the components and the presentation rate, such that large frequency differences and fast presentation rates produce the most SS (Miller \& Heise, 1950; Shonle \& Horan, 1976; van Noorden, 1975, Note 2). However, the perception is also influenced by the higher order variables of the listener's attentional set (van Noorden, 1975) and the total composition of the sequence (McNally \& Handel, 1977).

There is evidence that SS can change the perceived temporal relationships within a sequence. Listeners have difficulty locating the time of occurrence of extraneous sounds presented in ongoing speech or music (Foder \& Bever, 1965; Gregory, 1978; Ladefoged \& Broadbent, 1960). As SS increases, the perception of temporal position or order for tones across streams can become more difficult (Bregman \& Campbell, 1971; Bregman \& Dannenbring, 1973; Bregman \& Rudnicky, 1975; Dannenbring \& Bregman, 1976a; van Noorden, 1975), and this effect is accentuated in untrained listeners (e.g., Warren, Obusek, Farmer, \& Warren, 1969). Dannenbring and Bregman (1976a) report that tones in different streams are judged to be overlapping in time, even when physically separate. Woods, Sorkin, and Boggs (1979) have shown that pitch segregation, a frequencydependent context effect, can degrade performance in a gap-discrimination task. Overall, these studies 
suggest that changes in the perceived temporal relations within a sequence under conditions of SS might be expected to influence gap discrimination.

\section{Gap Discrimination with Tonal Markers}

Our primary concern in relating SS and gap discrimination is the effect of marker frequency on gap discrimination. Many studies using two-tone sequences have shown that performance is strongly affected by frequency separations within a marker pair (Collyer, 1974; Divenyi, 1971; Divenyi \& Danner, 1977; Divenyi \& Hirsh, 1972; Divenyi \& Sachs, 1978; Kinney, 1961; Perrott \& Williams, 1971; van Noorden, 1975, Note 1; Williams, Elfner, \& Howse, 1979; Williams \& Perrott, 1972). With all other parameters held constant, gap thresholds increase monotonically as a function of the difference between the marker frequencies. This frequency effect increases with marker duration, except for durations of $3 \mathrm{msec}$ or less, in which the stimuli have broad spectra (Williams et al., 1979; Williams \& Perrott, 1972). Similar effects are obtained with three-tone sequences (Williams \& Elfner, 1976).

Only a few investigators (Kinney, 1961; Fitzgibbons, Pollatsek, \& Thomas, 1974; van Noorden, 1975, Note 1) have examined gap discrimination for longer sequences, although such sequences would seem most likely to exhibit the effects, if any, of SS. In agreement with the results for short sequences, these studies found that performance deteriorated with increasing frequency separation between sequence components. Van Noorden (Note 1) reported that there were no discontinuities in the gap-threshold functions associated with the boundary where the frequency separation became large enough to change the percept from one stream to two. In most cases, however, this boundary region is itself subject to variation, which would obscure any discontinuities in the functions.

Although Fitzgibbons, Pollatsek, and Thomas (1974) and van Noorden (Note 1) used a range of frequency disparities sufficient to vary SS, no measures of SS were obtained. A later study by van Noorden (1975) did compare detectable displacements of tones in continuous sequences with measurements of the temporal coherence boundary obtained with similar sequences. ${ }^{1} \mathrm{He}$ found that performance continued to deteriorate with increasing frequency differences up to and beyond the temporal coherence boundary.

The present series of experiments examines gap discrimination and judgments of SS for tonal sequences expected to vary in perceptual organization. Because two-tone sequences are least subject to possible effects of SS (Norman, 1967; van Noorden, 1975), the results for two-tone sequences will be used as a reference to evaluate performance for longer sequences. Two-tone sequences are also used most fre- quently for gap discrimination measures of temporal processing in the peripheral auditory system (e.g., Abel, 1972; Divenyi \& Danner, 1977). Our goal is to determine whether SS plays an important role in limiting performance in gap discrimination conditions that involve large frequency differences between markers.

\section{EXPERIMENT 1}

This experiment measured training effects using two-tone sequences that form the core of longer sequences used in later experiments.

\section{Method}

Listeners. Three normal-hearing female listeners were paid for their participation. They were tested 2 h daily, 5 days a week over a period of $31 / 2$ months. None had had previous experience in any psychoacoustic experiment.

Apparatus and Stimuli. The sequences were composed of sinusoids generated by two Tektronix (FG501) function generators with onset/offset ramps determined by two Grason-Stadler (1287B) electronic switches. Intensity was controlled by four HewlettPackard (350D) attenuators and a Crown (D60) amplifier. The tones were presented diotically over TDH-39 earphones mounted in Grason-Stadler (001) cushions to listeners seated in soundtreated chambers (IAC 1200A). Presentation of the stimuli and recording of the data were controlled by a PDP-11/34 computer.

Seven two-tone sequences were constructed, with frequency differences between the tones equal to $1 / 8,1 / 4,1 / 2,1,11 / 2,2$, and $21 / 2$ octaves around a geometric mean of $1,600 \mathrm{~Hz}$. This range corresponds to differences of $139-3,959 \mathrm{~Hz}$. The higher tone was always presented first. All tones were $100 \mathrm{msec}$ in duration, measured at the half-voltage points, with 5-msec onset/offset ramps. The stimuli were presented at $80 \mathrm{~dB}$ SPL except for the lowfrequency tones for the 2 - and $21 / 2$-octave conditions. The latter were reduced $10 \mathrm{~dB}$ to equate relative loudness.

Procedure. Thresholds were estimated using a two-interval forced-choice (2IFC) procedure. The tone sequences in the two observation intervals differed only in the presence or absence of a gap, which could appear in either interval with equal probability. The listeners indicated the interval in which the gap occurred and were given feedback concerning the correct response. When no gap was inserted, the offset of the first tone and the onset of the second overlapped at the half-voltage points. The time between the two observation intervals was $350 \mathrm{msec}$. The time between the last listener's response and the beginning of the next trial was $600 \mathrm{msec}$. After randomizing the presentation order of the seven markerfrequency conditions, 10 or 1180 -trial blocks were completed for each condition to assure asymptotic performance.

For each frequency difference between markers, four gapduration increments $(\Delta t)$ were chosen, equally spaced on a logarithmic scale, such that performance ranged from $55 \%$ to $95 \%$ correct. The order of presentation for the four $\Delta$ ts was randomized, with the restriction that each value occur equally often within an 80 -trial block. The $\Delta$ t required for $\mathrm{d}^{\prime}=1.0$, the gap threshold, was estimated from a linear psychometric function fitted to the data on $\mathrm{d}^{\prime}$-by-log $\Delta \mathrm{t}$ coordinates. For our $2 \mathrm{IFC}$ procedure, $\mathrm{d}^{\prime}=1.0$ corresponded to $75 \%$ correct. The fits were generally good to excellent $(r \geqslant .85)$. Occasionally, gap thresholds estimated by this procedure for individual trial blocks were markedly different from the median. Threshold estimates more than three times larger or smaller than the median for each listener and condition were discarded, and logs of the remaining estimates were used to obtain the geometric-mean gap threshold for that condition and values for \pm 1 standard deviation (SD) around the mean. Average gap 
thresholds across listeners were also geometric or log means. This screening and data-reduction procedure was applied consistently across all gap-discrimination experiments reported below. In Experiment 1 , screening resulted in the loss of less than $1 \%$ of the data, and no estimates were discarded from the final four trial blocks.

\section{Results and Discussion}

Figure 1 illustrates the effects of training on performance for the seven marker-frequency conditions. Mean performance across the first three trial blocks is compared with that for the last four trial blocks for each listener and for the average across listeners. Functions for the middle trial blocks are omitted for clarity, since performance remained essentially unchanged from the fourth block to the end of training. Although training serves to reduce gap thresholds for all conditions and stabilize performance, a strong frequency effect remains. Gap thresholds across the final four trial blocks range from $5.89 \mathrm{msec}$ for the smallest frequency difference to $24.21 \mathrm{msec}$ for the largest.

Specific comparisons with other gap-discrimination data are difficult because of differences in parameters. The most comparable data are those of Williams and Perrott (1972) for 100-msec markers. They obtained gap thresholds of $24 \mathrm{msec}$ for their largest frequency difference (roughly $3 / 4$ octave), while the present study obtained a value of about $9 \mathrm{msec}$. Divenyi and Danner (1977) tested larger frequency differences, but they used much shorter $(20-\mathrm{msec})$

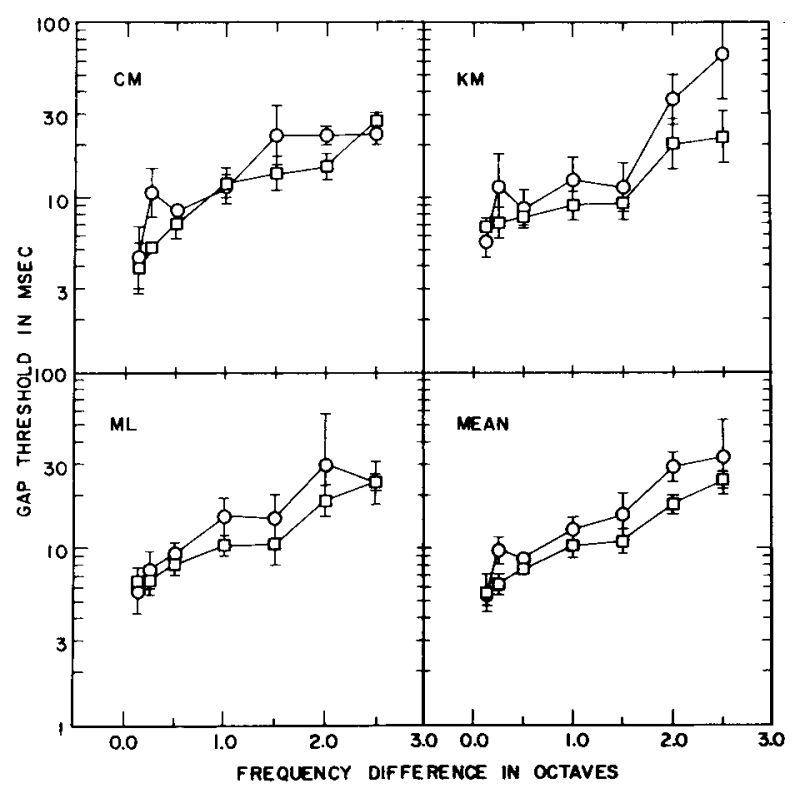

Figure 1. Performance for two-tone sequences across the first four blocks of trials (circles) compared with that across the last four trial blocks (squares). The data points are geometric means. The error bars indicate ratios of 1 SD around the mean. Error bars reflect variability across replications in panels 1,2 , and 3 , and variabiltiy across listeners in panel 4 . markers. Their gap thresholds for a 2-octave condition were as low as $8 \mathrm{msec}$, while we obtained a value of $18 \mathrm{msec}$ for that frequency difference.

Williams and Elfner (1976), using idealized gapthreshold functions for the two-tone sequences obtained by Williams and Perrott (1972), have attempted to quantify the relation between marker duration and the frequency difference beyond which gap discrimination deteriorates. Their break point for $100-\mathrm{msec}$ markers occurs at frequency differences smaller than our 1/8-octave condition, or well within a "critical band" centered at 1,600 Hz (Scharf, 1970; Zwicker, Flottorp, \& Stevens, 1957). Williams and Elfner propose that spectral overlap of the two markers is necessary for best performance and that a break in the gap-threshold function occurs when the markers no longer overlap. Although this describes the data for small frequency differences moderately well, it does not explain why performance continues to deteriorate with increasing frequency differences. Our gap thresholds increase monotonically throughout the range tested, which exceeds 12 critical bandwidths. We would expect to see very little interaction in the peripheral auditory system, as measured in forward masking, for example, between markers separated by 2 or $2 \frac{1 / 2}{2}$ octaves. Yet gap thresholds continue to increase in this range. Only one listener (K.M.) shows signs of reaching a plateau in performance.

\section{EXPERIMENT 2}

This experiment examines gap discrimination in four-tone sequences similar to those used by Fitzgibbons et al. (1974). The present study used a wider range of frequency differences and a procedure that allowed gap thresholds to be estimated for all conditions.

\section{Method}

Apparatus and Stimuli. Two function generators and electronic switches supplemented the equipment from Experiment 1. Seven four-tone sequences were constructed by adding a tone both before and after each two-tone sequence from Experiment 1 . The frequency differences between the second and third tones thus ranged from $1 / 8$ to $2 \frac{1}{2}$ octaves $(139$ to $3,959 \mathrm{~Hz}$ ), with the higher tone presented first. The frequencies of the additional components, the first and fourth tones in each sequence, were within the range defined by the middle components. The first was $3 \%$, or $1 / 24$ octave, lower than the second, and the fourth was $3 \%$, or $1 / 24$ octave, higher than the third. This allowed us to assess gap discrimination for small and large frequency differences within the same sequence and made the tones within the high- and lowfrequency pairs perceptually more distinct. All tones were $100 \mathrm{msec}$ in duration, with 5-msec onset/offset ramps as in Experiment 1.

Procedure. Two complete sets of data were collected, using a 2IFC procedure. In the "no-gap," or standard, interval in the first set, the offset of the preceding tone overlapped the onset of the next at the half-voltage points, as in Experiment 1 . In the standard interval in the second set, a 10 -msec gap between tones, measured at half-voltage points, eliminated spectral overlap. The listener indicated the sequence in which the longer gap occurred. For con- 
venience, we refer to the first as a detection task and the second as a discrimination task.

As in Fitzgibbons et al. (1974), gaps could be placed between the first and second, second and third, or third and fourth tones in a four-tone sequence. A "minimal uncertainty psychophysical procedure" (Watson, Kelly, \& Wroton, 1976) was used to assess gap discrimination. One of the seven four-tone sequences and one of the three gap positions were randomly selected and tested for four successive 80-trial blocks. The remaining two gap positions were tested for that sequence before another sequence was selected. To minimize uncertainty further, every trial block began with two practice trials which contained clearly audible 200 -msec gaps at the target position. The screening procedure discarded $7 \%$ of the data.

\section{Results and Discussion}

Figure 2 compares performance for conditions using 0 -msec vs. 10 -msec standard intervals. The leftmost point on each function shows performance averaged across all of the $3 \%$-frequency differences for gaps inserted in the first or last position within a sequence. The small, but significant, differences in performance for these gap positions (Neff, 1979) are not important for the present discussion. The remainder of each function traces performance for the second gap position, where marker frequencies differed by $1 / 8$ to $2 \frac{1}{2}$ octaves. As in the two-tone sequences, gap thresholds increase with increasing frequency difference. A repeated-measures analysis of variance (ANOVA) was done on a $\log$ transform of the data for the center gap position. The 1/24-octave data were omitted to avoid confounding the effect of gap position with that of frequency difference. As is evident from Figure 2, there is a highly significant effect of marker-frequency difference $[F(6,12)=14.41$, $\mathrm{p}<.001]$. In addition, gap thresholds are consis-

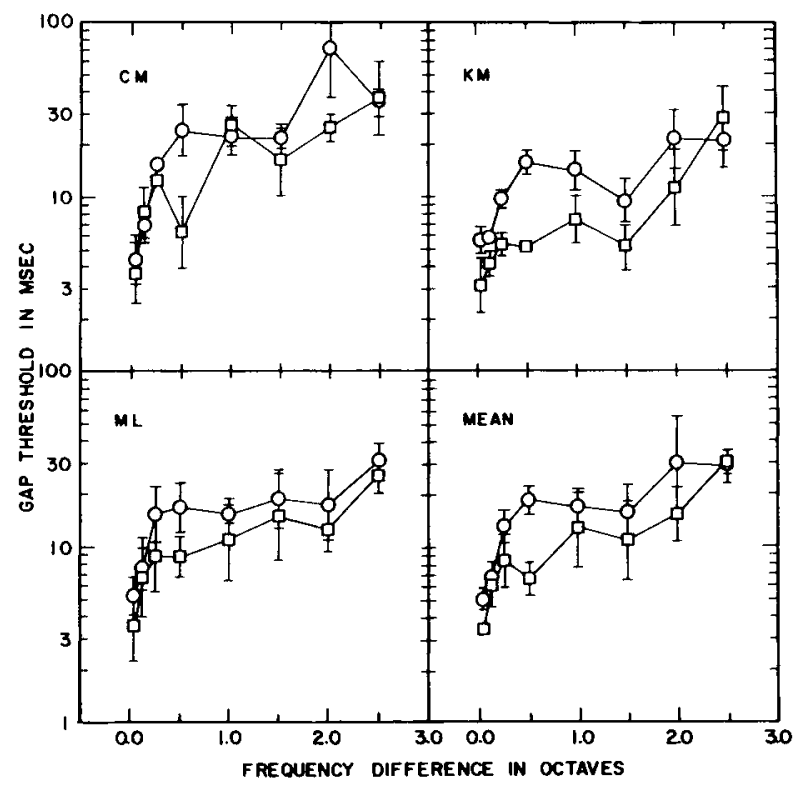

Figure 2. Gap thresholds for conditions using 0-msec standard intervals (circles) and 10-msec standard intervals (squares). tently lower for discrimination than detection conditions $[F(1,2)=51.60, p<.02]$. The slopes of the functions for the two tasks differ sufficiently to yield a significant task $\times$ frequency difference interaction $[F(6,12)=4.33, p<.02]$. Several studies have shown that gap thresholds generally increase with longer standard intervals (Abel, 1972; Divenyi \& Danner, 1977; Divenyi \& Sachs, 1978; Penner, 1976). Our observation of better performance for discrimination is therefore unexpected, since both the elimination of possible spectral cues and the use of a longer standard interval should raise gap thresholds. For standard intervals similar to ours, however, Divenyi and Sachs (1978) also find better performance with the longer duration. ${ }^{2}$ Our discrimination data were collected after those for detection, so the lower gap thresholds could simply reflect continued practice with the four-tone sequences. However, large practice effects seem unlikely, since the listeners had been trained to asymptote for the two-tone sequences. Given the significant task effect, detection and discrimination data will be considered independently in later comparisons across experiments.

Fitzgibbons et al. (1974) assessed gap detection with four-tone sequences containing either a frequency difference of roughly $21 / 2$ octaves or $1 / 4$ octave. A gap threshold for the $1 / 4$-octave condition could not be estimated from their data. For the $21 / 2-$ octave condition, however, their gap threshold of $20 \mathrm{msec}$ (for $\mathrm{d}^{\prime}$ of approximately 1.0 ) is comparable to our estimates of 30 and $28 \mathrm{msec}$, given the variability for this condition.

\section{EXPERIMENT 3}

In this experiment, judgments of SS were obtained for sequences expected to vary in perceptual organization. These same sequences were then used in the final gap-discrimination task, Experiment 4. The presentation rate and the frequency separation of the tones within a sequence were varied parametrically to induce various degrees of SS (van Noorden, 1975, Note 2). The fastest sequences with the largest frequency differences were expected to cause the strongest perception of SS.

\section{Method}

Apparatus and Stimuli. A D-A converter (DEC AR-11) interfaced to the computer allowed the output of a single Tektronix (FG501) function generator to present more than four frequencies in rapid succession. Otherwise, the equipment was identical to that described earlier.

Sequences were composed of 4200 -msec tones, 8 100-msec tones, or $1650-\mathrm{msec}$ tones, which will be referred to as $4-200$, 8-100, or 16-50 sequences, respectively. The three presentation rates combined changes in both number of tones and tone duration, such that all the sequences were of equal length. The frequency composition of the four-tone sequences was identical to that described for Experiment 2. Each 8-100 or 16-50 sequence was 
generated by repeating the appropriate four-tone sequence. Onset/ offset ramps were $5 \mathrm{msec}$ in duration and the tones were separated by $5 \mathrm{msec}$ between half-voltage points.

Procedure. Because SS can be strongly influenced by the listener's attentional set (van Noorden, 1975), written instructions and a visual representation of SS were given to each listener before data collection began. They were instructed to press the first button on the response box if they could perceive the sequence as a single temporally coherent unit or the second button if the sequence seemed to break apart into two or more perceptually discrete units. In van Noorden's terms, these instructions should produce an attentional set in which the listener tries to maintain the percept of a single sequence as long as possible. If one assumes that perceiving a tone sequence as a single temporally coherent stream facilitates gap discrimination, then our instructions should have encouraged the same attentional set for judgments of SS as was implicit in the gap-discrimination task to follow.

The 21 conditions ( 7 frequency sets $\times 3$ presentation rates) occurred five times in random order within each 105-trial block. Twenty blocks were completed, with presentation order randomized independently for each block, yielding a total of $100 \mathrm{judg}$ ments for each condition. The sequences were each $800 \mathrm{msec}$ long with $600 \mathrm{msec}$ between the end of the response interval and the start of the next sequence. The listeners were given no feedback.

\section{Results and Discussion}

Responses indicating the perception of SS were tabulated for each listener across trials. The percentage of SS votes for individual listeners and means across listeners for each condition are presented in Figure 3. As in Figure 2, the abscissa reflects the maximum frequency difference within the sequences. Error bars are omitted from the average data for clarity, because the large individual differences are

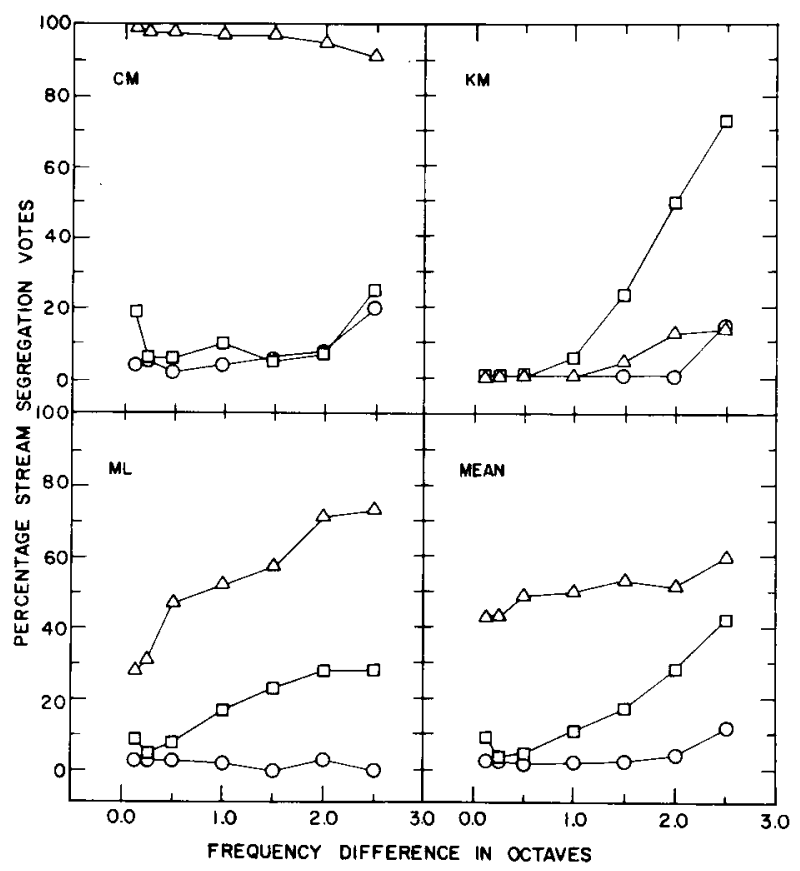

Figure 3. Streaming judgments obtained for sequences composed of 4200 -msec (circles), 8100 -msec (squares), and 1650 -msec (triangles) tones. Panel 4 contains arithmetic means across listeners. evident. As shown in Figure 3, the percentage of streaming votes either remained constant or increased for increasing frequency differences within the sequences. The pattern of votes as a function of presentation rate, however, was unique for each listener. The average response pattern is presented, despite the large differences between individuals, because that pattern typifies the responses expected given the work of van Noorden (1975).

To quantify the differences in response patterns, a repeated-measures ANOVA was done for each listener. The data were converted to proportions and an arcsin transformation was applied (Walker \& Lev, 1953). Listener C.M.'s responses are dominated by the effect of presentation rate $[F(2,399)=2302.81$, $\mathrm{p}<.001$, which accounts for over $90 \%$ of the variance. The interaction of frequency difference and presentation rate was also significant $[F(12,399)=$ $4.42, p<.001$ ] but accounted for only $1 \%$ of the variance. There was no significant effect of frequency difference alone. Listener K.M. showed a completely different pattern. There were significant effects of frequency difference $[F(6,399)=49.48, p<.001]$, presentation rate $[\mathrm{F}(2,399)=72.36, \mathrm{p}<.001]$, and the interaction of the two $[\mathrm{F}(12,399)=19.72, \mathrm{p}<.001]$. The variances accounted for by these factors were $27 \%, 13 \%$, and $22 \%$, respectively. K.M.'s data do not agree with van Noorden $(1975,1977$, Note 2), who found that increasing the presentation rate facilitated streaming. For K.M., the sequences of intermediate rate received the highest percentage of streaming votes. Listener M.L. also showed significant effects of frequency difference $[F(6,399)=11.89, p<$ $.001]$, presentation rate $[F(2,399)=206.26, p<.001]$, and the interaction of the two $[\mathrm{F}(12,399)=5.28, \mathrm{p}<$ .0011 . However, her response pattern typifies that expected by van Noorden, with the most streaming votes obtained for the fastest sequences with the largest frequency differences. The effect of presentation rate dominates the ratings, accounting for over $43 \%$ of the variance, while the other two factors combined account for only $15 \%$. The average data resemble those for Listener M.L.

The results of this experiment demonstrate the difficulties inherent in obtaining ratings of stream segregation. Despite considerable efforts to structure the listening situation, each listener's response pattern was unique. Most studies of SS use larger numbers of listeners and simple stimuli, such as continuously alternating cycles of two sinusoids which differ in frequency. We were unable to take this approach because our goal was to relate SS data to gap-discrimination data collected under similar conditions. Even under optimum conditions, however, considerable intra- and interlistener variability is reported (e.g., Bregman, 1978c; Bregman \& Dannenbring, 1973; Dannenbring \& Bregman, 1976a; Miller \& Heise, 
1950; Shonle \& Horan, 1976). Of course, the more variability associated with the rating data, the more difficult it is to relate judgments of SS to performance in tasks such as gap discrimination, which show little variability.

\section{EXPERIMENT 4}

In this experiment, 12 of the sequences from Experiment 3 were used in a gap-discrimination task in order to compare ratings of SS and gap-discrimination performance. Several conditions employed $100-\mathrm{msec}$ tone durations, as did earlier experiments, so the effect of embedding gaps in progressively longer sequences could also be examined.

\section{Method}

Apparatus and Stimuli. The equipment was identical to that used in Experiment 2. The 12 sequences included maximum frequency differences of $1 / 8,1 / 2,1 \frac{1}{2}$, and $2 \frac{1}{2}$ octaves and 4-200, 8-100, or 16-50 presentation rates.

Procedure. A 2IFC procedure was used with a $10-\mathrm{msec}$ standard interval, that is, the discrimination task of Experiment 2. Due to large individual differences in performance, a set of six $\Delta$ ts were chosen for each condition instead of the four used in previous experiments. The order of presentation of the six $\Delta$ ts was randomized, with the restriction that each value occur equally of ten within a 120-trial block. Listener C.M. was tested individually on some conditions using only four $\Delta$ ts to enable a complete set of data to be collected before she quit working. Gap thresholds were calculated as described earlier. Screening eliminated $8 \%$ of the data.

As in Experiment 2, gaps could occur in three positions within the four-tone sequences. For the 8- or 16-tone sequences, gaps were inserted only within the final four-tone segment and the listeners were informed of this constraint. Pilot work had indicated that gaps in the final portion of longer sequences introduced changes in rhythm between the last four tones and the ones preceding. Listeners could choose the correct interval simply by attending to the rhythm change. To eliminate this salient cue, brief variable-length silent intervals were inserted between each group of four tones. The durations used were drawn randomly without replacement from the six $\Delta$ ts available for each trial block, with the two listening intervals independently randomized. Introduction of silent intervals ranging from 2 to $128 \mathrm{msec}$ eliminated the rhythm cue and caused no apparent difference in SS.

Gaps could be inserted in three positions within the final four tones, as described for Experiment 2. One of the 12 sequences ( 4 frequency sets $\times 3$ presentation rates) and one of the three gap positions were randomly selected, and three successive 120-trial blocks were completed. The remaining two gap positions were tested for that sequence before the next sequence was selected. As in Experiment 2, every block began with two practice trials, which contained 200 -msec gaps.

\section{Results and Discussion}

Figure 4 compares performance for the three presentation rates. As in Figure 2, the leftmost point on each function shows gap thresholds averaged across all the $3 \%$, or $1 / 24$-octave, frequency differences. There were no significant changes in performance for these small differences. The remainder of each function shows gap thresholds for frequency differences ranging from $1 / 8$ to $2 \frac{1}{2}$ octaves. Regardless of pre-

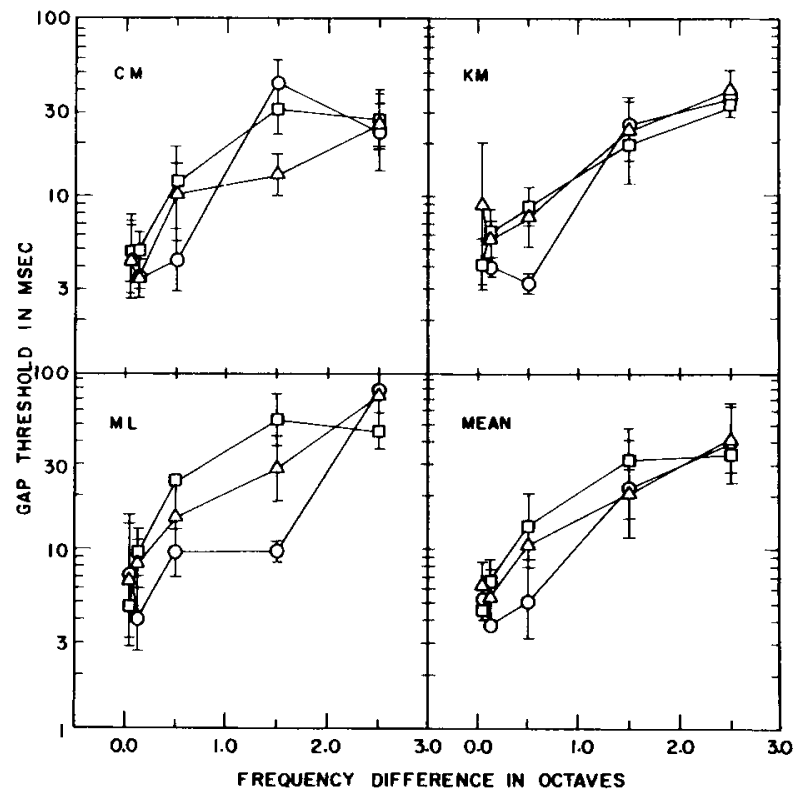

Figure 4. Gap discrimination data for 10 -msec standard intervals obtained for the same sequences of 4200 -msec (circles), 8 100 -msec (squares), and 1650 -msec (triangles) tones presented in Figure 3.

sentation rate, performance deteriorates as the frequency difference increases. A repeated-measures ANOVA was done on the log transform of these data, omitting the 1/24-octave differences. The frequency effect was highly significant $[F(3,12)=35.65$, $p<.001]$ and accounted for over $55 \%$ of the total variance. The effect of both presentation rate and the interaction of frequency difference and presentation rate were nonsignificant. Presentation rate accounted for only .1\% of the total variance.

The sequences of Experiment 4 involved changes in both tone duration and number of tones per sequence. The anticipated increase in gap thresholds as a function of number of tones presented could have been counterbalanced by a decrease in gap thresholds as a function of marker duration. To assess the effect of changing marker duration alone, a set of control conditions was completed using 200-, $100-$, or 50 -msec markers in two-tone sequences for four frequency differences. The procedure was identical to that of Experiment 1, except for the use of a 10 -msec standard interval. Figure 5 presents the average data for K.M. and M.L.; C.M. did not participate. A repeated-measures ANOVA showed no significant effect of marker duration, although the effect of marker frequency was again highly significant $[F(3,3)=147.54, p<.001]$. Thus, it seems unlikely that changes in marker duration could have played a significant role in Experiment 4.

Comparisons of similar conditions across the three gap-discrimination experiments allow us to examine 


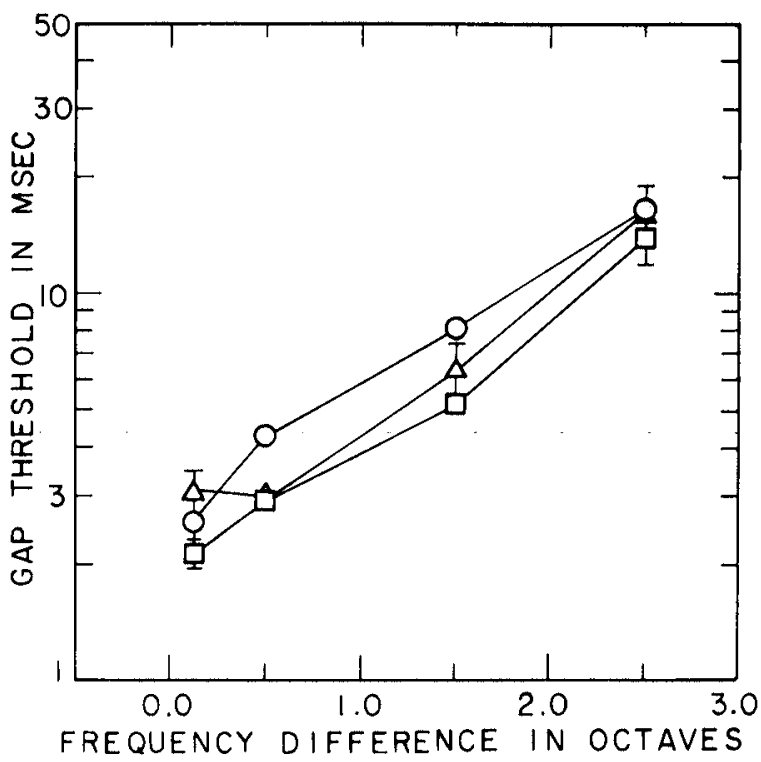

Figure 5. Mean gap thresholds for two-tone sequences with tone durations of $200 \mathrm{msec}$ (circles), $100 \mathrm{msec}$ (squares), and $50 \mathrm{msec}$ (triangles).

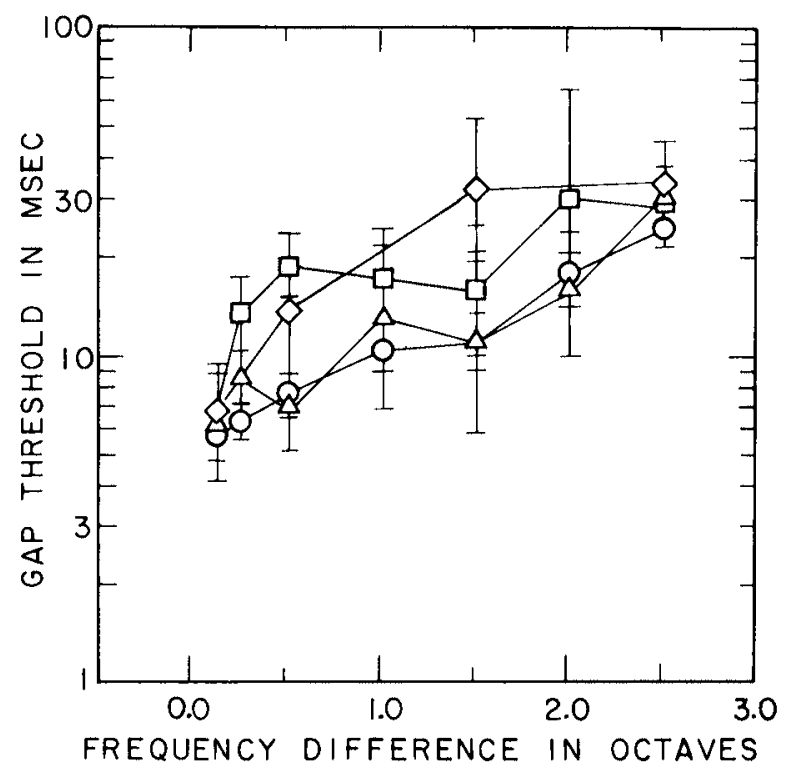

Figure 6. Mean gap thresholds for 100 -msec tone durations for the two-tone sequences of Experiment 1 (circles), the four-tone sequences of Experiment 2 for 0-msec (squares) and 10-msec (triangles) standard intervals, and the eight-tone sequences of Experiment 4 (diamonds).

the effect of sequence length for tones of constant duration. Figure 6 presents data for $100-\mathrm{msec}$ tone durations from the two-tone sequences of Experiment 1 , the four-tone sequences of Experiment 2 for both 0 - and 10-msec standard intervals, and the eighttone sequences of Experiment 4. Regardless of sequence length, gap thresholds increase as the fre- quency difference increases. In a repeated-measures ANOVA, this effect was highly significant $[\mathrm{F}(3,6)=$ $46.87, p<.001]$ and accounted for over $64 \%$ of the total variance. The change in gap thresholds as a function of sequence length is not significant. There is a highly significant interaction of frequency difference with sequence length $[F(9,18)=7.28, p<.001]$, but it accounts for only $9 \%$ of the total variance. Performance depends primarily on the frequencies of the two tones directly bounding the gap, regardless of sequence length.

Again, these data emphasize the dominant effect of marker-frequency difference in determining gap thresholds. The data also suggest that gap discrimination may not be related to SS in any direct way, because gap discrimination is not influenced by presentation rate. In contrast, the effects of presentation rate and the frequency $\times$ presentation rate interaction on SS are well documented in the literature (e.g., Bregman, 1978d; van Noorden, 1975, 1977, Note 2) and have been demonstrated in Experiment 3. Van Noorden (1975, 1977, Note 2) has shown that the listener's attentional set, a concept implying central processes, can influence the effect of presentation rate. It seems plausible to propose that gap discrimination is limited primarily by peripheral processes, whereas SS involves both peripheral and central components.

\section{GENERAL DISCUSSION}

The present series of experiments used discrete tonal sequences, highly trained listeners, and a test procedure for the gap-discrimination tasks in which there was no uncertainty about where in the sequence a gap might occur. For these conditions, SS does not appear to be the primary factor limiting gapdiscrimination performance as the frequency difference between markers increases.

It is difficult to explore the role of SS in basic psychoacoustic tasks such as gap discrimination without some compromises in experimental design. Our discrete sequences were not ideal for the production of SS, but were much longer than the sequences used in most gap-discrimination tasks. Although we were not able to use exactly the same sequences in Experiments 3 and 4, we do not believe (based on judgments by the authors and verbal reports from the listeners) that the introduction of brief gaps in Experiment 4 changed the pattern of SS that we measured in Experiment 3. Brief gaps have little influence on SS in continuous sequences when the durations of the tones are brief. Dannenbring and Bregman (1976b) have shown that for tone durations of $100 \mathrm{msec}$ or less, a 100-msec gap, the largest gap they tested, did not change the percept from two streams to one. Regardless of the influence of brief gaps, we cannot be 
sure that SS was actually manipulated in the gapdiscrimination task at the point in the sequences where the gaps were inserted. Listeners may have based their SS judgments on the whole sequence in Experiment 3 but attended to only the last four tones when asked to make gap-discrimination decisions in Experiment 4. Even if SS did not occur at the ends of the longest sequences, however, this would not invalidate the major conclusion that the effect of marker frequency in gap discrimination is not dependent on the existence of SS. The frequency effect is very robust and occurs with all the sequences to about the same degree. If the phenomenon of SS is not so robust, this provides further evidence that SS is not directly responsible for the effect of marker frequency in gap discrimination. We believe gap thresholds and SS judgments are related in that they are both affected by the frequency separation between tones, presumably due to frequency analysis in the peripheral auditory system. They differ in that only SS judgments are affected by presentation rate, perhaps due to processing at higher levels. The question of why gap thresholds continue to increase with increasing markerfrequency difference remains unresolved, but this effect does not appear to be due to stream segregation.

\section{REFERENCE NOTES}

1. van Noorden, L. P. A. S. Discrimination of time intervals bounded by tones of different frequencies. Instituut voor Perceptie Onderzoek Annual Progress Report, 1971, 6, 12-15.

2. van Noorden, L. P. A. S. Rhythmic fission as a function of tone rate. Instituut voor Perceptie Onderzoek Annual Progress Report, 1971, 6, 9-12.

\section{REFERENCES}

ABet, S. M. Discrimination of temporal gaps. Journal of the Acoustical Society of America, 1972, 52, 519-524.

Breaman, A. S. Asking the 'what for' question in auditory perception. In M. Kubovy \& J. Pomerantz (Eds.), Perceptual organization. Hillsdale, N.J: Erlbaum, 1978. (a)

Bregman, A. S. Auditory streaming: Competition among alternative organizations. Perception \& Psychophysics, 1978, 23, 391 398. (b)

Bregman, A. S. Auditory streaming is cumulative. Journal of Experimental Psychology, 1978, 4, 380-387. (c)

Bregman, A. S. The formation of auditory streams. In J. Requin (Ed.), Attention and performance VII. Hillsdale, N.J: Erlbaum, 1978. (d)

Bregman, A. S., \& Campbell, J. Primary auditory stream segregation and perception of order in rapid sequences of tones. Journal of Experimental Psychology, 1971, 89, 244-249.

Bregman, A. S., \& Dannenbring, G. L. The effect of continuity on auditory stream segregation. Perception \& Psychophysics, 1973, 13, 308-312.

Bregman, A. S., \& Rudnicky, A. I. Auditory segregation: Stream or streams. Journal of Experimental Psychology: Human Perception and Performance, 1975, 1, 263-267.

Collyer, C. E. The detection of a temporal gap between two disparate stimuli. Perception \& Psychophysics, 1974, 16, 96-100.

Dannenbring, G. L., \& Bregman, A. S. Stream segregation and the illusion of overlap. Journal of Experimental Psychology: Human Perception and Performance, 1976, 2, 544-555. (a)

Dannengring, G. L., \& Bregman, A. S. Effect of silence between tones on auditory stream segregation. Journal of the Acoustical Society of America, 1976, 59, 987-989. (b)

DivenYI, P. L. The rhythmic perception of micromelodies: Detectability by human observers of a time increment between sinusoidal pulses of two different, successive frequencies. In E. Gordon (Ed.), University of Iowa studies in the psychology of music (Vol. 7). Iowa City: University of Iowa Press, 1971.

Divenyi, P. L., \& DANNer, W. F. Discrimination of time intervals marked by brief acoustic pulses of various intensities and spectra. Perception \& Psychophysics, 1977, 21, 125-142.

Divenyi, P. L., \& Hirsh, I. J. Discrimination of the silent gap in two-tone sequences of different frequencies. Journal of the Acoustical Society of America, 1972, 52, 166. (Abstract)

Divenyi, P. L., \& SAchs, R. M. Discrimination of time intervals bounded by tone bursts. Perception \& Psychophysics, 1978, 24, 429-436.

Fitzgibbons, P. J., Pollatsek, A., \& Thomas, I. B. Detection of temporal gaps within and between tonal groups. Perception \& Psychophysics, 1974, 16, 522-528.

Foder, J. A., \& Bever, T. G. The psychological reality of linguistic segments. Journal of Verbal Learning and Verbal Behavior, 1965, 4, 414-420.

Gregory, A. H. Perception of clicks in music. Perception \& Psychophysics, 1978, 24, 171-174.

KINNEY, J. A. S. Discrimination in auditory and visual patterns. American Journal of Psychology, 1961, 74, 529-541.

Ladefoged, P., \& Broadbent, D. E. Perception of sequence in auditory events. Quarterly Journal of Experimental Psychology, $1960,12,162-170$.

MCNALly, K. A., \& HANDEL, S. Effect of element composition on streaming and the ordering of repeating sequences. Journal of Experimental Psychology: Human Perception and Performance, 1977, 3, 451-460.

Miller, G. A., \& Heise, G. A. The trill threshold. Journal of the Acoustical Society of America, 1950, 22, 637-638.

NEFF, D. L. Gap discrimination as a function of the structure of the tonal sequence. Unpublished master's thesis, University of Nebraska at Omaha, 1979.

Norman, D. A. Temporal confusions and limited capacity processors. Acta Psychologica, 1967, 27, 293-297.

Penner, M. J. The effect of marker variability on the discrimination of temporal intervals. Perception \& Psychophysics, 1976, $19,466-469$.

Perrott, D. R., \& Williams, K. N. Auditory temporal resolution: Gap detection as a function of interpulse frequency disparity. Psychonomic Science, 1971, 25, 73-74.

Scharf, B. Critical bands. In J. V. Tobias (Ed.), Foundation of modern auditory theory (Vol. 1). New York: Academic Press, 1970.

Shonle, J. I., \& HoRAN, K. E. Trill threshold revisited. Journal of the Acoustical Society of America, 1976, 59, 469-471.

van Noorden, L. P. A. S. Temporal coherence in the perception of tone sequences. PhD thesis, Technische Hogeschool, Eindhoven, The Netherlands, 1975.

van Noorden, L. P. A. S. Minimum differences of level and frequency for perceptual fission of tone sequences ABAB. Journal of the Acoustical Society of America, 1977, 61, 10411045.

W alken, H. M., \& Lev, J. Statistical inference. New York: Holt, Rinehart \& Winston, 1953.

WARren, R. M., ObuSeK, C. J., FARMer, R. M., \& W WRren, R. P. Auditory sequence: Confusion of patterns other than speech and music. Science, 1969, 164, 586-587.

Watson, C. S., Kelly, W. J., \& Wroton, H. W. Factors in the discrimination of tonal patterns. II. Selective attention and learning under various levels of stimulus uncertainty. Journal 
of the Acoustical Society of America, 1976, 60, 1176-1186.

Williams, K. N., \& ElfNeR, L. F. Gap detection with three auditory events: A single-channel process. Journal of the Acoustical Society of America, 1976, 60, 423-428.

Williams, K. N., Elfner, L. F., \& Howse, W. R. Auditory temporal resolution: Effects of sensation level. Journal of Auditory Research, 1979, 19, 265-270.

Williams, K. N., \& Perrott, D. R. Temporal resolution of tonal impulses. Journal of the Acoustical Society of America, 1972, 51, 644-647.

Woods, D. D., Sorkin, R. D., \& Boggs, G. J. Stimulus context and duration discrimination. Perception \& Psychophysics, 1979, 26, 127-132.

Zwicker, E., Flottorp, G., \& Stevens, S. S. Critical bandwidth in loudness summation. Journal of the Acoustical Society of America, 1957, 29, 548-557.

\section{NOTES}

1. The temporal coherence boundary (van Noorden, 1975) marks the transition from the percept of one stream to two or more, when the listener is trying to hear coherence.

2. Divenyi and Sachs (1978) define their standard durations from the onset of one marker to the onset of the next, whereas we define our times from the offset of the first marker. Our values of 0 and $10 \mathrm{msec}$ correspond to times of 7.5 and 17.5 in their terms. We compare these data to Divenyi and Sachs' 10- and 20-msec data.

(Manuscript received December 24, 1980; revision accepted for publication January 16,1982 .) 